Reprod. Nutr. Dévelop., 1981, 21 (2), 185-192.

\title{
Mécanisme d'action du nouvel antibiotique kirromycine
}

\author{
par G. SANDER (1)
}

Laborafoire de Biochimie, Ecole polyfechnique (Laboratoire associé no 240 du CNRS), 91128 Palaiseou Cedex.

Summary. Mechanism of action of the new antibiotic, kirromycin.

The discovery and the elucidation of the mechanism of action of a new family of antibiotics is described. These antibiotics, here represented by kirromycin, all inhibit bacterial protein synthesis by acting on the protein elongation factor Tu, blocking its release from the ribosome during the elongation cycle and thereby inhibiting peptide bond formation. A number of these compounds have been successfully used as feed additives.

\section{Introduction.}

En 1972-1973, trois groupes différents ont publié indépendamment la découverte de trois nouveaux antibiotiques produits par Streptomyces ef présentant des propriétés spectrales analogues (Wolf ef Zähner, 1972 ; Vos, 1972 ; Berger et al., 1973). Il s'agis-

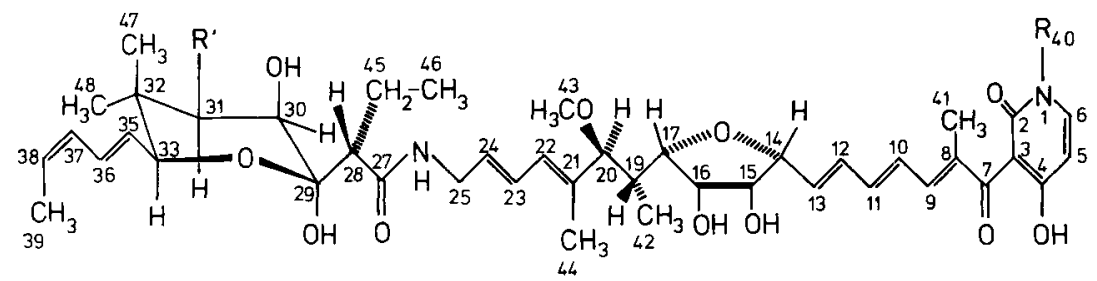

$$
\begin{array}{llc} 
& & \text { calcd M.W. } \\
\text { Mocimycin (Kirromycin) } R=\mathrm{H}^{\prime} \mathrm{R}^{\prime}=\mathrm{OH} & \mathrm{C}_{43} \mathrm{H}_{60} \mathrm{~N}_{2} \mathrm{O}_{12} & 797 \\
\text { Aurodox } \mathrm{R}=\mathrm{CH}_{3} \mathrm{R}^{\prime}=\mathrm{OH} & \mathrm{C}_{44} \mathrm{H}_{62} \mathrm{~N}_{2} \mathrm{O}_{12} & 811 \\
\text { Efrotomycin } \mathrm{R}=\mathrm{CH}_{3} ; \mathrm{R}^{\prime}=\text { Disaccharide } & \mathrm{C}_{59} \mathrm{H}_{88} \mathrm{~N}_{2} \mathrm{O}_{20} & 1145 \\
\text { Heneicomycin } \mathrm{R}=\mathrm{CH}_{3} ; \mathrm{R}^{\prime}=\mathrm{H} & \mathrm{C}_{44} \mathrm{H}_{62} \mathrm{~N}_{2} \mathrm{O}_{11} & 795
\end{array}
$$

FIG. 1. - La structure chimique de la kirromycine et d'autres antibiotiques apparentés

(Parmeggiani et Sander, 19800).

(1) Present address : Max-Planck-Institut für Molekulare Genetik, Ihnestrasse 63-73, D-1000 Berlin 33 (Dahlem), R.F.A. 
saif de produits colorés en jaune, faiblement acides, et pratiquement insolubles dans l'eau, mais formant des sels alcalins solubles dans l'eau. Dans une série de travaux, Maehr et al. (1980) ont ensuite élucidé la structure chimique de deux de ces produits, l'aurodox ef la mocimycine. La structure de cette dernière a été établie indépendamment par Vos et Verwiel (résumé dans Maehr et al., 1980) ; la kirromycine est très probablement identique à la mocimycine (bibliographie détaillée : Parmeggiani et Sander, 1980). Par la suite, d'autres antibiotiques appartenant à ce groupe, comme l'efrotomycine et l'heneicomycine (fig. 1), ont encore été isolés.

Le mode d'action de la kirromycine a été élucidé entre 1974 et 1980 principalement dans le laboratoire de Parmeggiani (pour une revue complète traitant de tous les aspects de l'action des antibiotiques de cette famille, voir Parmeggiani et Sander, 1980).

\section{Matériel et méthodes.}

Tous les essais ont été exécutés en utilisant un système in vitro provenant d'E. Coli ef dont les composants essentiels sont les suivants :

1) Les ribosomes. Ceux-ci doivent être lavés et débarrassés de toute activité enzymatique contaminante ;

2) Les facteurs d'élongation (EF-Tu, EF-Ts, EF-G) purs, sans aucune contamination avec des GTPases non spécifiques ;

3) L'aminoacyl-tARN, représenté par le phénylalanyl-tARN (Phe-tARN) ;

4) L'acide polyuridylique utilisé comme ARN messager (Parmeggiani et Sander, 1981 ; Sander et al., 1975 ; Chinali, Wolf et Parmeggiani, 1977).

\section{Résultats.}

Mécanisme de l'élongation. - L'addition d'un acide aminé à une chaîne polypeptidique en formation (élongation) comporte les étapes suivantes : (I) fixation du «complexe ternaire» EF-Tu.GTP.aa-łARN au site «A $)$ du ribosome (fig. $2 b$ ). Bien que l'aa-tARN puisse être lié au ribosome même en absence d'EF-Tu et de GTP, son positionnement correct dans le site $A$ requiert, dans les conditions optimales pour la synthèse protéique (5-7 $\mathrm{mM} \mathrm{Mg}^{2+}$ ), la formation de ce complexe. Le facteur EF-Tu ne peut complexer l'aa-tARN que s'il a préalablement fixé une molécule de GTP (dans le schéma de la fig 2 cette conformation active est visualisée par la forme globulaire du facteur). (II) Hydrolyse du GTP en GDP et $P_{i}$ qui aboutit à la formation du complexe EF-Tu.GDP et s'accompagne d'un changement conformationnel profond. Le complexe EF-Tu.GDP n'est plus capable d'interagir avec l'aa-tARN et quitte le ribosome. La conformation inactive (EF-Tu.GDP) est visualisée schématiquement par la forme carrée de la molécule (fig. 2c). (III) Formation de la liaison peptidique. Tant qu'il reste lié au ribosome, le facteur Tu empêche la formation de la liaison peptidique, probablement en tenant l'aa-tARN à l'écart de la « peptidyl transférase », centre ribosomique qui exécute cette réaction (fig. 2). Après la libération d'EF-Tu. GDPं, la liaison peptidique se forme spontanément entre l'aa-tARN lié au site $A$ ef le peptidyl-tARN lié au site «P» (fig. $2 c$ ). (IV) «Translocation»: l'interaction entre un complexe 


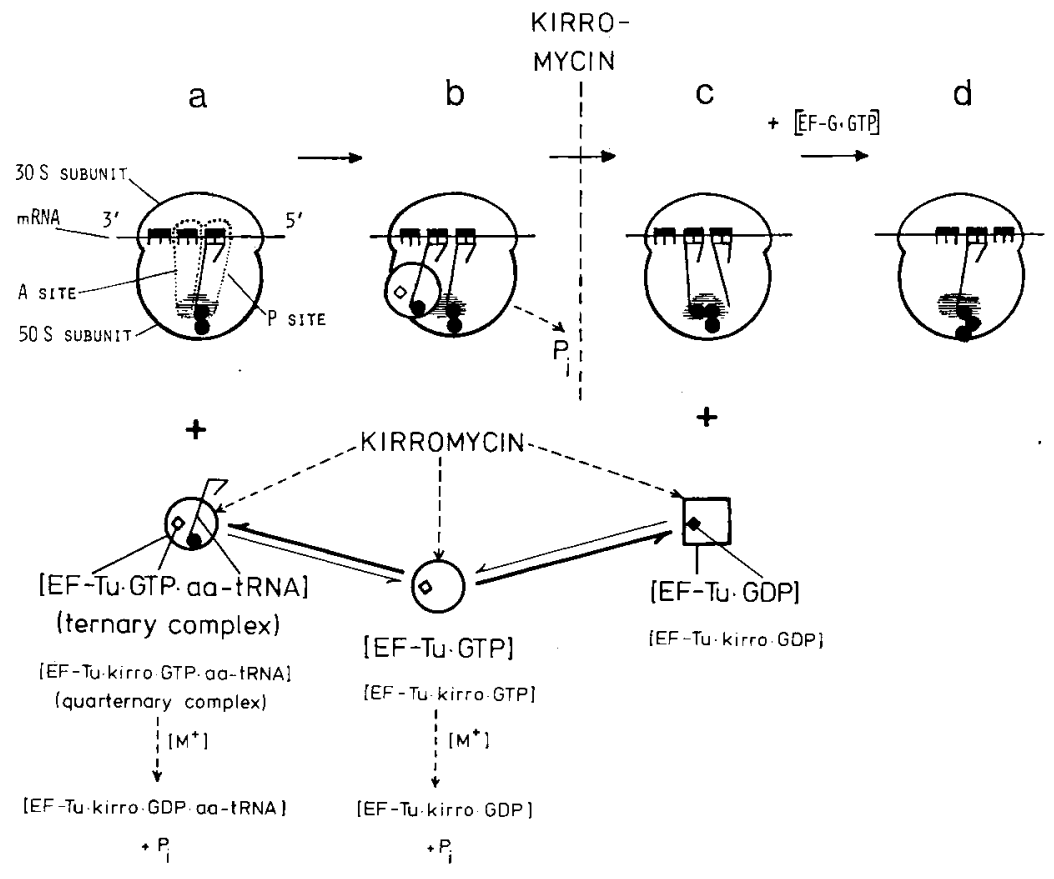

FIG. 2. - Le cycle d'élongation d'une chaine polypeptidique ef son inhibition par la kirromycine. Pour l'action du facteur EF-Ts, omise dans le schéma, voir le texte. EF-Ts et la kirromycine ne peuvent pas se lier simultanément au facteur Tu.

EF-G.GTP et le ribosome catalyse le déplacement du peptidyl-tARN du site $A$ au site $P$; ceci est accompagné de l'éjection du tARN qui a été déchargé au cours de la formation de la liaison peptidique. (V) Hydrolyse du GTP, réaction qui occasionne la libération d'EF-G.GDP. Le ribosome est alors prêt pour un nouveau cycle d'élongation. (VI) Recyclage d'EF-Tu.GDP. L'affinité du facteur Tu pour GDP est environ 500 fois plus élevée que son affinité pour GTP $\left(\mathrm{K}_{\mathrm{eq}}^{\prime \mathrm{GDP}}=1 \mathrm{nM}, \mathrm{K}_{\mathrm{eq}}^{\prime \mathrm{GTP}}=500 \mathrm{nM}\right)$. Le facteur Ts intervient pour permettre la formation d'une faible quantité du complexe EF-Tu. GTP qui reconnaît l'aa-tARN. L'affinité du Tu pour le GTP est alors multipliée par $\sim 500$.

Action de la kirromycine dans les conditions de la synthèse protéique in vitro (Wolf, Chinali ef Parmeggiani, 1974, 1977 ; Parmeggiani, Wolf et Chinali, 1976 ; Chinali, Wolf et Parmeggiani, 1977 ; Parmeggiani et Sander, 1980). - La kirromycine forme un complexe $1: 1$ avec EF-Tu, que ce facteur soif libre ou complexé avec GDP ou GTP; la kirromycine et le facteur EF-Ts s'excluent mutuellement. L'affinité d'EF-Tu.GTP pour l'antibiotique est 40 fois plus importante que celle d'EF-Tu. GDP $(0,05 \mu \mathrm{M}$ vs. $2 \mu \mathrm{M}$, Fasano et al., 1978). En présence d'aa-tARN et de GTP, il se forme un complexe quaternaire EF-Tu.kirromycine.GTP.aa-tARN qui se fixe au ribosome (fig. $2 b$ ) et y reste accroché malgré l'hydrolyse du GTP. Par conséquent, l'aa-tARN ne peut pas réagir avec le peptidyl-tARN (voir plus haut, réaction III) ef toutes les réactions suivantes sont bloquées. 
Il semblerait donc que le changement conformationnel produit au cours de la réaction EF-Tu.GTP $\rightarrow$ EF-Tu.GDP n'a pas lieu en présence de kirromycine; de plus l'antibiotique induit la formation d'un complexe entre EF-Tu et aa-tARN non seulement en présence de GTP, mais également avec du GDP ou même en absence de tou nucléotide. Ce complexe est capable de se lier au ribosome.

Action de la kirromycine sur la réaction GTPasique d'EF-Tu (Wolf, Chinali et Parmeggiani, 1974, 1977 ; Parmeggiani, Wolf ef Chinali, 1976 ; Parmeggiani et al., 1977 ; Chinali, Wolf et Parmeggiani, 1977 ; Fischer et al., 1977 ; Fasano et al., 1978 ; Sander ef al., 1979, 1980 ; Parmeggiani et Sander, 1980.) - La réaction GTPasique d'EF-Tu qui est normalement associée à la liaison du complexe ternaire au ribosome, est inhibée, dans le système complet, après l'hydrolyse d'une molécule de GTP par molécule d'EF-Tu (en présence d'un excès de ribosomes). Cette réaction a été étudiée en détail en omettant le facteur $\mathrm{G}$.

Le résultat principal est que le facteur Tu est le porteur du centre catalytique de cette réaction, puisque le complexe kirromycine-EF-Tu est capable de catalyser une activiłé GTPasique type turnover en absence de tout autre effecteur biologique (fig. 3 ,

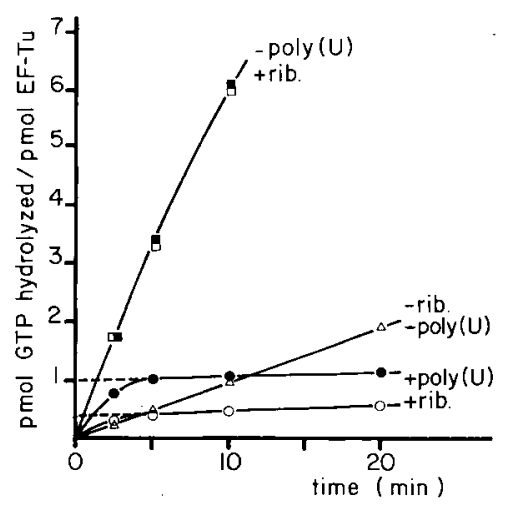

\section{FIG. 3. - L'influence de la kirromycine sur la GTPase d'EF-Tu.}

12 pmol d'EF-Tu, 200 pmol de Phe-tRNAPhe, 450 pmol de $\left[\gamma-{ }^{32} \mathrm{P}\right] \mathrm{GTP}$ et $5 \mathrm{nmol}$ de kirromycine, avec $(0, \bullet)$ ou sans $(\Delta, \square, \quad) 10 \mu \mathrm{g}$ d'acide polyuridylique, dans $225 \mu \mathrm{l}$ de $60 \mathrm{mM}$ Tris- $\mathrm{HCl}(\mathrm{pH} \mathrm{7,8)}$ contenant $10 \mathrm{mM}$ $\mathrm{MgCl}_{2}, 30 \mathrm{mM} \mathrm{KCl}, 30 \mathrm{mM} \mathrm{NH}_{4} \mathrm{Cl}$ ef $2 \mathrm{mM}$ dithiothréitol. (๑, a) préincubation (10 min à $0^{\circ} \mathrm{C}$ ) avec GTP, mais sans EF-Tu ; réaction déclenchée par l'addition d'EF-Tu; $(0, a)$ préincubation $\left(10 \mathrm{~min}\right.$ à $\left.0^{\circ} \mathrm{C}\right)$ avec EF-Tu, mais sans GTP; réaction déclenchée par l'addition du GTP (modifié d'après Parmeggiani, Wolf et Chinali (1976).

$\Delta)$. A part son effet inhibiteur, la kirromycine agit donc comme inducteur de GTPase (voir aussi fig. 2, partie basse). Qui plus est, en absence de messager, cette activité est spécifiquement stimulée par l'aa-tARN, le ribosome et encore plus par la combinaison de ces 2 composants (fig. 3, $\mathbf{0},-)$ ). Lorsque l'on ajoute également du messager, le complexe quaternaire EF-Tu.kirromycine.GTP.aa-łARN est bloqué sur le ribosome après l'hydrolyse d'une seule molécule de GTP (fig. 3, •), comme dans le système complet pour la synthèse de protéines. Ceci est dû au fait que le facteur, une fois lié au ribosome, ne peut plus interagir avec le GTP (fig. 3, o). Dans cette expérience on a d'abord fixé le facteur sous forme du complexe EF-Tu.kirromycine.aa-tARN (en absence de nucléotides) à un excès de mRNA. ribosomes. Dans les conditions choisies, l'efficacité de cette réaction représente 60 p. 100 de celle obtenue en présence de GTP. Si le GTP est alors ajouté, on constate que seuls les 40 p. 100 du facteur Tu non encore complexés aux ribosomes sont capables d'hydrolyser ce GTP. Par conséquent, l'interaction entre codon et anticodon entraîne la fixation du complexe EF-Tu.kirromycine sur le ribosome et empêche son interaction avec du GTP libre. 
La présence de la kirromycine favorise la formation du complexe EF-Tu. GTP. - Comme décrit plus haut, le complexe EF-Tu.GDP est normalement $\sim 500$ fois plus stable que le complexe EF-Tu.GTP. Or, la présence de la kirromycine déplace la constante d'équilibre entre EF-Tu et GTP vers des valeurs presque égales à celles qu'on trouve normalement pour EF-Tu. GDP, c'est-à-dire $1 \mathrm{nM}$ environ (tabl. 1). En effet, nous avons

\section{TABLEAU 1}

Constantes d'équilibre pour les complexes EF-Tu.GTP/GDP : influence de la kirromycine d $0^{\circ} \mathrm{C}$ (Fasano et al., 1978)

\begin{tabular}{lc}
\hline \multicolumn{1}{c}{ Système } & \multicolumn{1}{c}{$\underline{K}^{\prime}(\mathrm{nM})$} \\
\hline EF-Tu.GTP. $\ldots \ldots \ldots \ldots \ldots \ldots \ldots \ldots \ldots$ & 590 \\
EF-Tu.GTP $+50 \mu M$ kirromycine $\ldots \ldots$ & 1,4 \\
EF-Tu.GDP $\ldots \ldots \ldots \ldots \ldots \ldots \ldots \ldots \ldots$ & 0,9 \\
EF-Tu.GDP $+50 \mu M$ kirromycine $\ldots \ldots$ & 0,8 \\
\hline
\end{tabular}

montré que l'antibiotique augmente de $\sim 12$ fois la vitesse d'association entre EF-Tu et GTP et diminue encore plus fortement, de $\sim 35$ fois, la vitesse de dissociation du complexe EF-Tu. GTP. Par contre, son effet sur les vitesses d'association et de dissociation entre EF-Tu et GDP est une légère augmentation des deux, ce qui ne change pas l'équilibre (Fasano et al. 1978 ; Parmeggiani ef al., 1978). Ceci est la condition préalable pour le « turnover » efficace de la réaction GTPasique qu'on observe en présence de la kirromycine.

Influence de sels monovalents. - Pour l'induction d'une activité GTPasique dépendante d'EF-Tu.kirromycine, la présence de cations monovalents est rigoureusement requise (Sander et al., 1979, 1980). Or, l'influence de cations monovalents sur l'association et la dissociation des complexes EF-Tu. GTP et EF-Tu. GDP est très modeste : ceci suggère que les cations monovalents interviennent au niveau de l'étape catalytique (Sander et al., 1979). Le ribosome supprime ce besoin, peut-être par l'apport des sels monovalents qui lui sont liés. Quel que soif le mécanisme réel, nos dernières expériences montrent que, comme les ribosomes, les sels monovalents augmentent sensiblement l'entropie d'activation pour la formation du complexe activé EF-Tu.GTP* (Bocchini ef al., 1980). Nous interprétons ce résultat comme indiquant une « ouverture » de la molécule d'EF-Tu par la kirromycine, ce qui augmenterait la chance d'une collision fructueuse par rapport à la conformation normale très fermée.

Mutants d'E. Coli possédant un facteur résistant à la kirromycine (Parmeggiani et Sander, 1980). - Cet organisme est normalement résistant à la kirromycine, parce que l'antibiotique ne peut pas franchir la barrière membranaire. Notre choix s'est donc porté sur des souches bactériennes dont la membrane est directement perméable aux antibiotiques (Fischer et al., 1977). D'autres auteurs ont perméabilisé la membrane de souches couramment utilisées par traitement à l'EDTA (Van de Klundert ef al., 1977). De plus, vu l'existence de deux gènes codant pour le facteur Tu, la résistance à la kirromycine implique que ces gènes soient tous deux altérés, puisque comme nous 
l'avons démontré, la sensibilité est dominante sur la résistance (Fischer et al., 1977). De fait, il a été démontré (Van de Klundert et al., 1977) qu'au cours de la sélection, un seul des gènes est inactivé et la modification de l'autre produit la résistance. Le facteur Tu résistant à la kirromycine a été caractérisé biochimiquement ; il montre, outre une résistance 300 fois plus élevée que le facteur sauvage, une activité GTPasique spontanée en absence de l'antibiotique (Fasano et Parmeggiani, 1980).

Applications. - Bien que tous les résultats décrits jusqu'ici aient été obłenus par l'intermédiaire d'un système in vitro d'E. Coli, cet organisme fait partie du grand nombre de bactéries ayant une résistance élevée contre les antibiotiques appartenant au groupe de la kirromycine. On a exploité la propriété de ces produits d'agir contre un nombre bien délimité de microorganismes, et d'être très peu toxiques pour les animaux, en les utilisant comme additif à la nourriture du bétail (tabl. 2). L'efficacité

TABLEAU 2

Efficacité de l'efrotomycine et de l'aurodox chez des animaux

(d'après Stutz et al., 1976 ; Marusich, Ogrinz et Mitrovic, 1974)

\begin{tabular}{|c|c|c|c|c|}
\hline \multirow[b]{2}{*}{ Efrotomycine (ppm) (1) } & \multicolumn{4}{|c|}{ Amélioration de } \\
\hline & $\begin{array}{l}\text { la croissance jour- } \\
\text { nalière moyenne } \\
\text { (p. 100) }\end{array}$ & $\begin{array}{l}\text { l'indice de } \\
\text { consommation } \\
\text { (p. 100) }\end{array}$ & $\begin{array}{l}\text { la } c \\
\text { journ }\end{array}$ & $\begin{array}{l}\text { onsommation } \\
\text { alière moyenne } \\
\text { (p. 100) }\end{array}$ \\
\hline $\begin{array}{r}2,5 \\
5,5 \\
11,0 \\
27,5\end{array}$ & $\begin{array}{l}6,2 \text { (NS) } \\
11,5 \\
15,0 \\
16,8\end{array}$ & $\begin{array}{l}4,0 \\
2,7 \text { (NS) } \\
8,4 \\
8,0\end{array}$ & & $\overline{-}$ \\
\hline 27,5 & 16,7 & 8,0 & & 10 \\
\hline \multicolumn{2}{|c|}{$\begin{array}{l}\text { Concentrations d'antibiotiques occasionnant des } \\
\text { améliorations comparables de la croissance journa- } \\
\text { lière moyenne et de l'indice de consommation }\left(^{2}\right) \ldots\end{array}$} & \multicolumn{3}{|c|}{$\begin{array}{l}\text { aurodox lincomycine bambermycines } \\
\text { (mg/g de nourriture) } \\
5,5-10 \quad 2,2-4,4\end{array}$} \\
\hline
\end{tabular}

(1) Stutz et al., 1976 (388 cochons). L'efrotomycine s'est également avérée très efficace, à $\geqslant 27,5$ ppm, pour combattre la dysentérie induite expérimentalement chez le porc (Foster et Harris, 1976).

( $\left.{ }^{2}\right)$ Marusich, Ogrinz et Mitrovic, 1974 (poulets, dindonneaux).

NS $=$ non significatif.

de l'aurodox, de l'efrotomycine et de la mocimycine se sont avérées comparables à celles trouvées pour d'autres additifs déjà utilisés commercialement. L'existence et la nature d'un lien éventuel entre l'action inhibitrice de la kirromycine chez $E$. coli et sa stimulation de la croissance chez les animaux supérieurs restent à être élucidées.

\section{Conclusion.}

Les antibiotiques de cette famille donnent un bon exemple de la possibilité d'une collaboration fructueuse entre les diverses disciplines scientifiques : entre 1972 et 1980 , non seulement la structure chimique d'un certain nombre d'entre eux a été établie, 
mais leur mécanisme d'action, à l'échelle moléculaire, a également éfé élucidé. Parallèlement, on en a amélioré les méthodes de production et on les a introduits dans la nourrifure animale. Ainsi ces antibiotiques sont maintenant parmi les mieux connus.

De plus, le fait qu'ils agissent non seulement comme inhibiteurs, mais également, par la suppression de mécanismes de contrôle allostérique, comme inducteurs d'une activité GTPasique, les a rendus essentiels pour la compréhension des mécanismes impliqués dans le fonctionnement normal de leur cible, le facteur d'élongation Tu.

6e Réunion du groupe Développement I.N.R.A., Clermont-Ferrand/Theix, 22-23 mai 1980.

\section{Références}

BERGER J., LEHR H. H., TEITEL S., MAEHR H., GRUNBERG E., 1973. A new antibiotic X-5108 of Streptomyces origin I. Production, isolation and properties. J. Antibiot., 26, 15-22.

BOCCHINI V., PARLATO G., DE VENDITTIS E., SANDER G., PARMEGGIANI A., 1980. Energetic aspects of the EF-Tu $\rightarrow$ dependent GTPase activity. A study using the antibiotic kirromycin Eur. J. Biochem., 113, 53-60.

CHINALI G., WOLF H., PARMEGGIANI A., 1977. Effect of kirromycin on elongation factor Tu. Location of the catalytic center for ribosome-elongation factor Tu GTPase activity on the elongation factor. Eur. J. Biochem., 75, 55-65.

FASANO O., BRUNS W., CRECHET J.-B., SANDER G., PARMEGGIANI A., 1978. Modification of elongation factor Tu.guanine nucleotide interaction by kirromycin. A comparison with the effect of aminoacyl-tRNA and elongation factor Ts. Eur. J. Biochem., 89, 557-565.

FASANO O., PARMEGGIANI A., 1980. The altered expression of the GTPase activity in a kirromycinresistant elongation factor Tu. Biochemistry (sous presse).

FISCHER E., WOLF H., HANTKE K., PARMEGGIANI A., 1977. Elongation factor Tu resistant to kirromycin in an Escherichia coli mutant altered in both fuf genes. Proc. nat. Acad. Sci. USA, 74, 4341 4345.

FOSTER A. G., HARRIS D. L., 1976. Efrotomycin, a drug for swine dysentery control. J. anim. Sci., 43, 252.

MAEHR H., LEACH M., WILLIAMS T. H., BLOUNT J. F., 1980. The chemistry of aurodox and related antibiotics. Can. J. Chem., 58, 501-526.

MARUSICH W. L., OGRINZ E. F., MITROVICM., 1974. A new antibiotic, X-5108, for improved growth abd feed conversion in poultry. Poultr. Sci., 53, 936-945.

PARMEGGIANI A., FASANO O., IVELL R., SANDER G., 1978. Properties of elongation factor Tu and mode of action of kirromycin, 349-356. In ZELINKA J., BALAN J., Ribosomes and nucleic acid metabolism, vol. 3, Publ. House Slovak Acad. Sci., Bratislava.

PARMEGGIANI A., FISCHER E., FASANO O., CRECHET J.-B., WOLF H., 1977. Studies on the mechanism of action of kirromycin and on the expression of elongation factor Tu resistance to this antibiotic, 178-182. In LEGOCKI A. B., Translation of natural and synthetic nucleotides, Publ. Poznan Agric. Univ., Poznan.

PARMEGGIANI A., SANDER G., 1980. Properties and action of kirromycin (mocimycin) and related antibiotics, 159-221. In SAMMES, P. G., Topics in antibiotic chemistry, Wiley \& Sons, Chichester, England.

PARMEGGIANI A., SANDER G., 1981. Properties and regulation of the GTPase activities of elongation factors Tu and $G$, and of initiation factor 2. Mol. cell. Biochem. (sous presse).

PARMEGGIANI A., WOLF H., CHINALI G., 1976. Mechanism of action of kirromycin, a new inhibitor of protein biosynthesis, 283-290. In ZELINKA J., BALAN J., Ribosomes and RNA Metabolism, vol. 2, Publ. House Slovak Acad. Sci., Bratislava.

SANDER G., IVELL R., CRECHET J.-B., PARMEGGIANI A., 1980. Interaction of elongation factor Tu with the riboomes. A study using the antibiotic kirromycin. Biochemistry, 19, 865-870. 
SANDER G., MARSH R. C., VOIGT J., PARMEGGIANI A., 1975. A comparative study of the 50S ribosomal subunit and several 50 S subparticles in EF-T- and EF-G-dependent activities. Biochemistry, 14, 1805-1814.

SANDER G., OKONEK M., CRECHET J.-B., IVELL R., BOCCHINI V., PARMEGGIANI A., 1979. Hydrolysis of GTP by the elongation factor Tu. kirromycin complex. Specific action of monovalent cations. FEBS Lett., 98, 111-114.

STUTZ M. W., JOHNSON S. L., OTTO K. G., MILLER B. M., 1976. Efrotomycin, a new growth permittant for swine. J. anim. Sci., 43, 259-260.

VAN DE KLUNDERT J. A.M., DEN TURK E., BORMAN A. H., VAN DER MEIDE P. H., BOSCH L., 1977. Isolation and characterization of a mocimycin resistant mutant of Escherichia coli with an altered elongation factor EF-Tu. FEBS Letf., 81, 303-307.

vos C., 1972. Antibiotic MYC 8003. Chem. Abstr., 77, 32742.

WOLF H., CHINALI G., PARMEGGIANI A., 1974. Kirromycin, an inhibitor of protein biosynthesis that acts on elongation factor Tu. Proc. nat. Acad. Sci. USA, 71, 4910-4914.

WOLF H., CHINALI G., PARMEGGIANI A., 1977. Mechanism of the inhibition of protein synthesis by kirromycin. Role of elongation factor Tu and ribosomes. Eur. J. Biochem., 75, 67-75.

WOLF H., ZÄHNER H., 1972. Stoffwechselprodukte von Mikroorganismen. 99. Mitteilung. Kirromycin. Arch. Mikrobiol., 83, 147-154. 